\title{
MODELACIÓN NUMÉRICA DE LA CIRCULACIÓN MARINA EN LAS BAHÍAS CALLAO Y MIRAFLORES
}

\section{NUMERICAL MODELING OF CIRCULATION IN CALLAO AND MIRAFLORES BAYS}

\author{
Mirian Centeno, Emanuel Guzmán y Paúl García \\ Grupo de Estudio de la Dinámica Marina, Ingeniería Mecánica de Fluidos, \\ Universidad Nacional Mayor de San Marcos, Lima 01, Perú
}

\section{RESUMEN}

El presente trabajo consiste en estudiar a nivel superficial la circulación marina en las bahías del Callao y Miraflores, mediante el uso del modelo numérico Princeton Ocean Model conocido como $\mathrm{POM}$, el cual es un conjunto de ecuaciones y parámetros que gobiernan la dinámica oceánica. El modelo POM se empleó con la finalidad de caracterizar el patrón de circulación en el área de estudio, analizando la influencia de los forzantes viento y marea (propagación del Norte) en la generación de corrientes marinas, así como los efectos que se producen a causa de la morfología costera y la presencia de la Isla; estos factores influyen en los resultados indicando una complejidad en las corrientes marinas, como la presencia de vórtices dentro de la bahía del Callao, así también la propagación de mareas se manifiestan en el área del puerto del Callao generando las corrientes de flujo y reflujo.

Conociendo la circulación marina en las Bahías de Callao y Miraflores, se podrá realizar estudios posteriores de dispersión de contaminante, descargas residuales, derrames accidentales de sustancias, así también estudios de transporte de sedimentos aportados por la presencia de los ríos Rímac y Chillón.

Palabras clave: Circulación marina, Princeton Ocean Model, Bahías del Callao y Miraflores

\section{ABSTRACT}

The present work is related to study the surface circulation in Callao and Miraflores bays using Princeton Ocean Model (POM) model, which is a set of equations and parameters that govern the ocean dynamics. POM was used to caracterize the circulation pattern in the study area, analyzing the main influence of wind stress and tide in the generation of currents and the effects occur because of the coastal morphology and the presence of the Island, these factors influence the results indicating a complexity in the ocean currents and the presence of vortices inside the bay of Callao, also tidal propagation manifests in the area of the port of Callao, generating currents ebb and flow.

In this way, knowing the ocean circulation in the Callao and Miraflores bays, further studies can be made pollutant dispersion, wastewater discharges, accidental spills of substances, and also sediment transport studies produced by the presence of Rimac and Chillon rivers.

Keywords: Marine Circulation, Princeton Ocean Model, Callao y Miraflores bays.

\section{INTRODUCCION}

Actualmente se requiere de estudios oceanográficos que nos permitan conocer la hidrodinámica en un área determinada, que pueden obtenerse de un análisis de datos recolectados durante inspecciones de campo, para luego ser interpretados; además de ello, estos estudios son complementados con una herramienta matemática conocida como los métodos numéricos que llevan al desarrollo de Modelos Numéricos, para nuestro caso el modelo numérico Princeton Ocean Model (POM), el cual es un conjunto de ecuaciones y parámetros que gobiernan la dinámica oceánica, es alimentado mediante datos de campo, así como datos de fuentes internacionales. 
En el presente trabajo se realizó la modelación numérica para conocer la circulación marina a nivel superficial en las bahías del Callao y Miraflores, analizando la influencia de los forzantes viento y marea (propagación del Norte) en la generación de corrientes marinas, así como los efectos que se producen a causa de la morfología costera y la presencia de la Isla San Lorenzo. (Ver Figura 1). El estudio se inicia con la recopilación y el procesamiento de los datos empleados que son descritos posteriormente, luego de ello se procede a trabajar con el modelo numérico, adecuando las condiciones de frontera y definiendo las características de la simulación. Finalmente los valores obtenidos de la modelación serán validados de manera cualitativa.

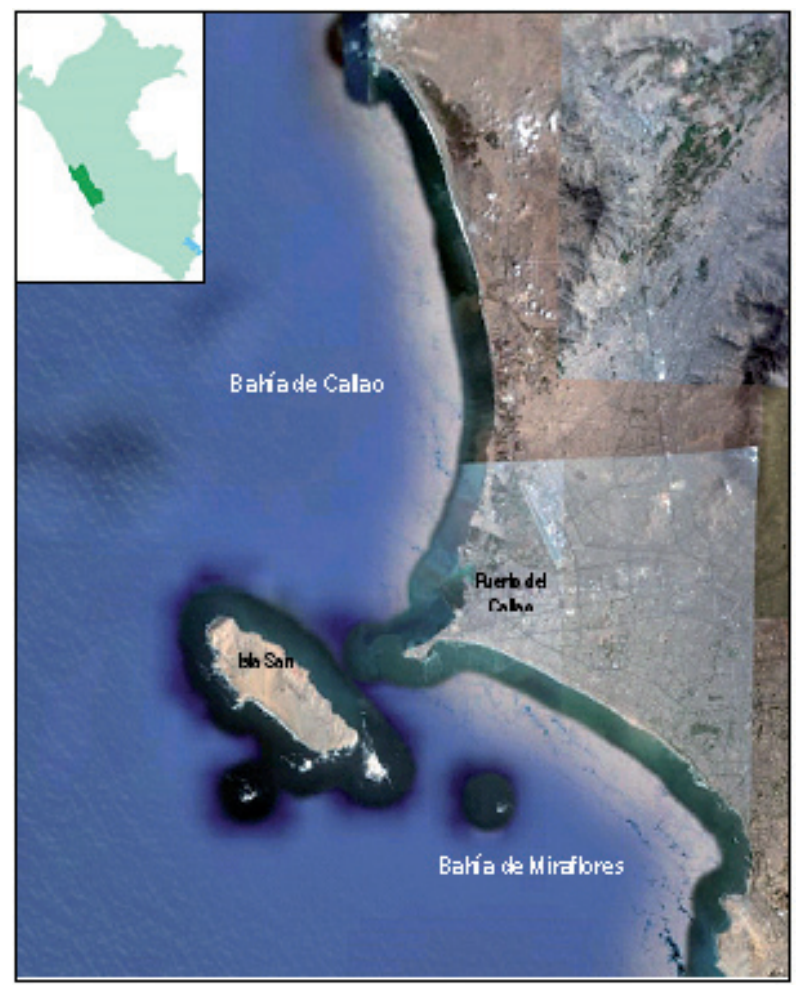

Figura 1. Área de estudio, Bahía del Callao y Miraflores, Lima - Perú.

\section{METODOLOGIA}

El desarrollo del presente trabajo se realizó en 2 etapas:

\section{a) Recopilación y Procesamiento de la información.}

En esta sección se recopilarán datos que son necesarios conocer para el desarrollo de la modelación numérica, los mismos que serán procesados en un formato adecuado e ingresados al modelo numérico.

\section{- Batimetría y perfil costero.}

Los datos de batimetría fueron obtenidos de la Carta HIDRONAV 2234, de la Dirección de Hidrografía y Navegación (DHN), para las Bahías del Callao y Miraflores, donde se puede observar que las isobatas están relacionadas a la presencia de la Isla San Lorenzo. El perfil costero para nuestra área de estudio en la bahía de Callao tiene una orientación Noroeste, para luego formar una especie de punta, continuando al Sur este perfil adopta una orientación Suroeste en la Bahía de Miraflores. El procesamiento de la batimetría se realizó mediante la digitalización de la carta, empleando el programa SURFER.

\section{- Vientos}

Los datos de vientos fueron obtenidos de la estación Chucuito ubicado en Latitud: $12^{\circ} 03^{\prime} 30^{\prime \prime} \mathrm{S}$ y Longitud: $77^{\circ} 09^{\prime} 00^{\prime \prime} \mathrm{W}$ de la $\mathrm{DHN}$, dicha estación cuenta con un registro de datos desde el año 1978 al 2004, para el trabajo se considero el promedio de los años 2000 al 2004, teniendo en cuenta que se está trabajando bajo condiciones normales, donde la magnitud de la velocidad oscila entre los valores de 3.5 a 6.5 nudos, con dirección predominante proveniente del Sur. El valor considerado promedio para trabajar el modelo numérico es viento de 6 nudos, dirección Sur.

\section{- Mareas}

Se dispone de información de Nivel medio del Mar (NMM), calculadas con el programa WXTIDE (desde 1970 a 2037), la cual proporciona información de NMM de las principales ciudades del mundo (más de 9500 estaciones repartidas por todo el mundo), cabe mencionar que este programa se puede descargar de manera gratuita en la siguiente dirección web: http://www.wxtide32. $\mathrm{com} /$. La estación de Callao se ubica en las siguientes coordenadas, Latitud: $12^{\circ} 02^{\prime} 00^{\prime \prime S}$ y Longitud: $77^{\circ} 09^{\prime} 00^{\prime \prime} \mathrm{W}$, donde se observa que el tipo de marea para nuestra área de estudio corresponde a un tipo de marea semidiurna.

Adicionalmente se cuenta con información de las 08 principales constantes armónicas de marea para Callao (Ubicación de la estación: Latitud: $12^{\circ} 02^{\prime} 00^{\prime \prime} S$, Longitud: $77^{\circ} 09^{\prime} 00^{\prime \prime} \mathrm{W}$ ), la cual fue extraída de la pagina web: http:// www.waterforecast.com/globaltide/ , que es un modelo que utiliza los datos de satélite del TOPEX/POSEIDON. (Ver Tabla 1) 
Tabla 1. Armónicos de marea. Bahía del Callao

\begin{tabular}{|c|c|c|}
\hline \multicolumn{3}{|c|}{ Tidal konstituents for: } \\
\hline Name & Amplitude & Phase \\
\hline M2 & 0.195 & 325.11 \\
\hline S2 & 0.076 & 332.63 \\
\hline K1 & 0.146 & 29.02 \\
\hline O1 & 0.06 & 351.69 \\
\hline N2 & 0.063 & 293.59 \\
\hline P1 & 0.047 & 25.47 \\
\hline K2 & 0.025 & 335.90 \\
\hline Q1 & 0.004 & 347.91 \\
\hline
\end{tabular}

\section{- Corrientes marinas}

Los datos de corrientes fueron obtenidos de los resultados del modelo Ocean Circulation and Climate Advanced Modelling (OCCAM), estos datos se encuentran en la pagina web http://www.noc.soton.ac.uk/JRD/OCCAM/ , en extensión NETCDF(Network Comman Data Format), para luego ser procesados mediante el programa FERRET(http://lpas.epfl.ch/MOD/ manuals/ferret/ ). Esta información nos servirá para tener un patrón de circulación en un área más amplia a nuestra zona de estudio.

Así también se esta considerando la hipótesis del patrón de circulación en las inmediaciones de la Isla San Lorenzo propuesto por PESI (Parsons Engineering Science International) en el Estudio de Factibilidad de una Planta de Tratamiento de aguas en Lima [1]. Ver Figura 2.

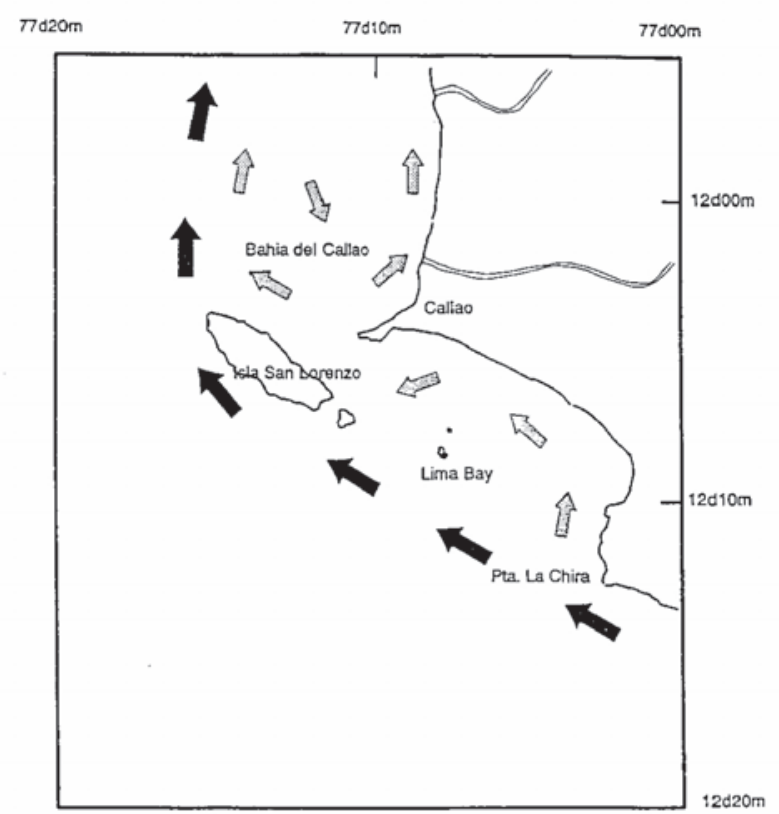

Figura 2. Hipótesis del patrón de circulación marina, propuesto por PESI (2000).
Del Estudio de Impacto Ambiental para el Diseño, Construcción, Operación y Cierre del Nuevo Terminal de Contenedores Adyacente al Rompeolas Sur del Terminal Portuario del Callao [2], se tiene un registro de las corrientes marinas a nivel superficial para la zona del puerto del Callao, ver Figura 3.

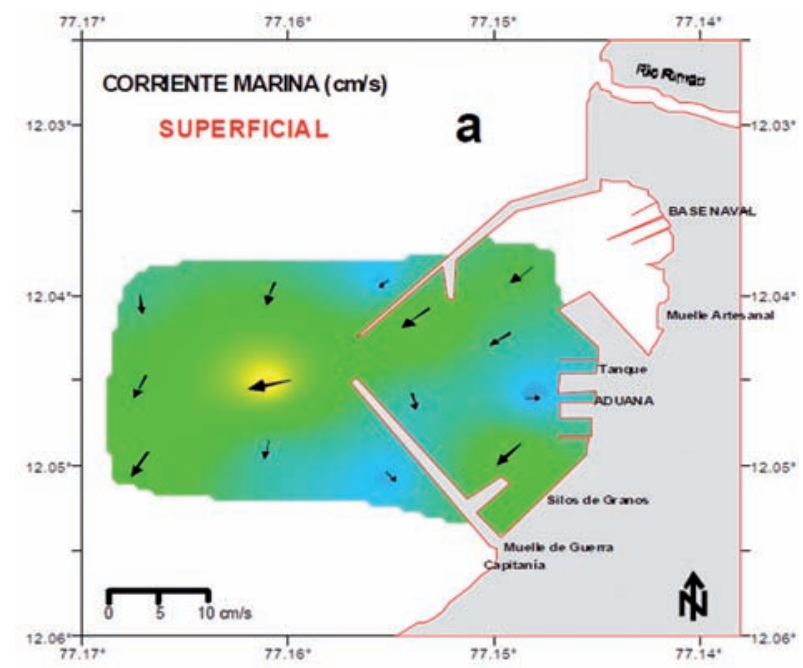

Figura 3. Corrientes marinas a nivel superficial, puerto del Callao.

En la Figura 4 se muestra una fotografía satelital de la NASA para Lima/Callo Área, Perú May 1997, donde se observa como el patrón de circulación arrastra consigo las descarga de desmontes para la bahía de Miraflores, y la descarga por parte del río Rímac en la bahía del Callao, mostrando las trazas de su trayectoria en dirección Norte.

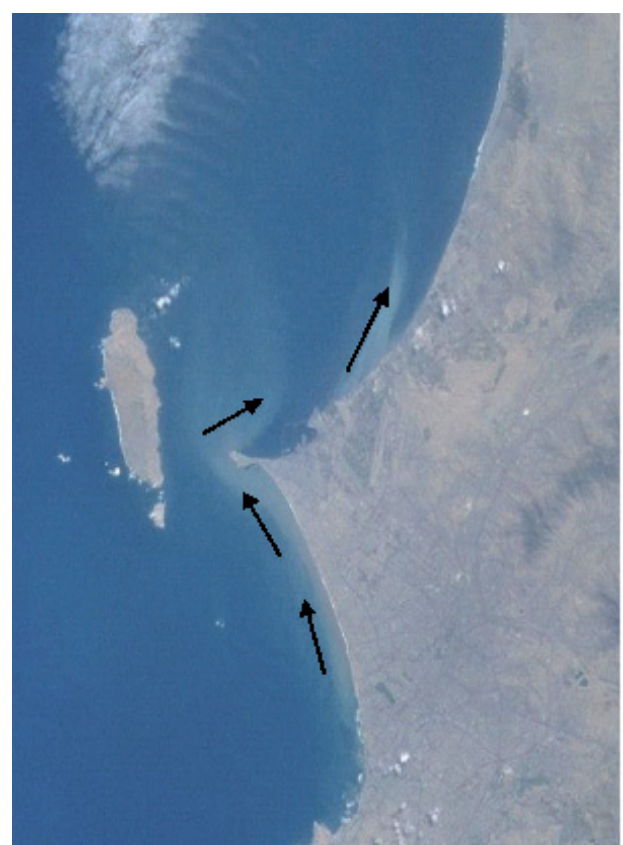

Figura 4. Fotografía satelital. NASA, Lima/Callo Área, Perú May 1997 


\section{- Propiedades del Agua de mar}

De acuerdo al boletín Oceanografía de las Aguas Costeras del Perú, Salvador Zuta y Oscar Guillen (1970) [3], se puede hablar de un agua costera con temperatura $<23^{\circ} \mathrm{C}$ como temperatura $(\mathrm{T})$ promedio en superficie, mientras para la salinidad (S) en el área del Callao, no se presentan variaciones estacionales; para el presente trabajo se esta considerando una temperatura de $22^{\circ} \mathrm{C}$, y un valor de salinidad de 35 UPS.

\section{b) Modelación Numérica}

Descripción de Modelo Numérico empleado (POM)

Se empleó el modelo numérico "Princeton Ocean Model" (POM), el cual es un modelo tridimensional de circulación oceánica basado en la integración numérica de las ecuaciones primitivas que rigen la dinámica de la circulación oceánica. El modelo fue desarrollado por Alan Blumberg y George L. Mellor en la década de los 70 en el programa de ciencias atmosféricas del Geophysical Fluid Dynamics Laboratory (GFDL) de los Estados Unidos de Norteamérica [4].

El código fuente (programa principal y subrutinas) está escrito en lenguaje de programación FORTRAN 77 y se puede trabajar en varios sistemas operativos como UNIX, LINUX. El modelo es de dominio público y su código fuente se encuentra disponible en la siguiente dirección web:

http://www.aos.princeton.edu.WWWPUBLIC/ htdocs.pom

Las ecuaciones que describen la circulación oceánica son una versión modificada de las ecuaciones de Navier-Stokes. Este sistema de ecuaciones cartesianas son transformadas a un sistema de coordenadas sigma, lo que permite representar de manera mas real el fondo marino, de acuerdo a ellos las ecuaciones básicas del modelo expresadas en coordenadas sigma son[5]:

Ecuación de continuidad:

$$
\frac{\partial \mathbf{D U}}{\partial \mathbf{x}}+\frac{\partial \mathbf{D V}}{\partial \mathbf{y}}+\frac{\partial \mathbf{w}}{\partial \boldsymbol{\sigma}}+\frac{\partial \eta}{\partial \mathbf{t}}=0
$$

Ecuaciones de la conservación del Momento Componente $\mathrm{x}$ :

$$
\begin{aligned}
& \frac{\partial \mathbf{U D}}{\partial \mathbf{t}}+\frac{\partial \mathbf{U}^{2} \mathbf{D}}{\partial \mathbf{x}}+\frac{\partial \mathbf{U V D}}{\partial \mathbf{y}}+\frac{\partial \mathbf{U} \mathbf{w}}{\partial \boldsymbol{\sigma}}-\mathbf{f V D}+\mathbf{g D} \frac{\partial \eta}{\partial \mathbf{x}}= \\
& \frac{\partial}{\partial \boldsymbol{\sigma}}\left(\frac{\mathbf{K}_{\mathbf{M}}}{\mathbf{D}} \frac{\partial \mathbf{U}}{\partial \boldsymbol{\sigma}}\right)-\mathbf{g} \frac{\mathbf{D}^{2}}{\boldsymbol{\rho}_{0}} \int_{\sigma}^{0}\left[\frac{\partial \rho}{\partial \mathbf{x}}-\left(\frac{\boldsymbol{\sigma}}{\mathbf{D}} \frac{\partial \mathbf{D}}{\partial \mathbf{x}} \frac{\partial \boldsymbol{\rho}}{\partial \boldsymbol{\sigma}}\right)\right] \mathbf{d} \boldsymbol{\sigma}+\mathbf{F}_{\mathbf{x}}
\end{aligned}
$$

Componente y:

$$
\begin{aligned}
& \frac{\partial \mathbf{V D}}{\partial \mathbf{t}}+\frac{\partial \mathbf{U V D}}{\partial \mathbf{x}}+\frac{\partial \mathbf{V}^{2} \mathbf{D}}{\partial \mathbf{y}}+\frac{\partial \mathbf{V w}}{\partial \boldsymbol{\sigma}}+\mathbf{f U D}+\mathbf{g D} \frac{\partial \eta}{\partial \mathbf{y}}= \\
& \frac{\partial}{\partial \boldsymbol{\sigma}}\left(\frac{\mathbf{K}_{\mathbf{M}}}{\mathbf{D}} \frac{\partial \mathbf{V}}{\partial \boldsymbol{\sigma}}\right)-\mathbf{g} \frac{\mathbf{D}^{2}}{\boldsymbol{\rho}_{0}} \int_{\sigma}^{0}\left[\frac{\partial \rho}{\partial \mathbf{y}}-\left(\frac{\boldsymbol{\sigma}}{\mathbf{D}} \frac{\partial \mathbf{D}}{\partial \mathbf{y}} \frac{\partial \boldsymbol{\rho}}{\partial \boldsymbol{\sigma}}\right)\right] \mathbf{d} \boldsymbol{\sigma}+\mathbf{F}_{\mathbf{y}}
\end{aligned}
$$

Componente z:

$$
\frac{1}{\mathbf{D}} \frac{\partial \mathbf{P}}{\partial \boldsymbol{\sigma}}=-\rho \mathbf{g} \mathbb{F}
$$

Conservación de la temperatura potencial

$$
\frac{\partial \theta \mathbf{D}}{\partial \mathbf{t}}+\frac{\partial \boldsymbol{\theta} \mathbf{U D}}{\partial \mathbf{x}}+\frac{\partial \boldsymbol{\theta} \mathbf{V D}}{\partial \mathbf{y}}+\frac{\partial \theta \mathbf{w}}{\partial \mathbf{z}}=\frac{\partial}{\partial \boldsymbol{\sigma}}\left(\frac{\mathbf{K}_{\mathbf{H}}}{\mathbf{D}} \frac{\partial \boldsymbol{\theta}}{\partial \boldsymbol{\sigma}}\right)+\mathbf{F}_{\Theta}
$$

Conservación de la salinidad

$$
\frac{\partial \mathbf{S D}}{\partial \mathbf{t}}+\frac{\partial \mathbf{S U D}}{\partial \mathbf{x}}+\frac{\partial \mathbf{S V D}}{\partial \mathbf{y}}+\frac{\partial \mathbf{S w}}{\partial \mathbf{z}}=\frac{\partial}{\partial \boldsymbol{\sigma}}\left(\frac{\mathbf{K}_{\mathbf{H}}}{\mathbf{D}} \frac{\partial \mathbf{S}}{\partial \boldsymbol{\sigma}}\right)+\mathbf{F}_{\mathbf{S}}
$$

Donde:

$$
\begin{aligned}
& \mathbf{F}_{\mathbf{x}}=\frac{\partial}{\partial \mathbf{x}}\left(2 \mathbf{A}_{M} \mathbf{D} \frac{\partial \mathbf{U}}{\partial \mathbf{x}}\right)+\frac{\partial}{\partial \mathbf{y}}\left[\mathbf{A}_{\mathbf{M}} \mathbf{D}\left(\frac{\partial \mathbf{U}}{\partial \mathbf{y}}+\frac{\partial \mathbf{V}}{\partial \mathbf{x}}\right)\right] \\
& \mathbf{F}_{\mathbf{y}}=\frac{\partial}{\partial \mathbf{y}}\left[\mathbf{A}_{\mathbf{M}} \mathbf{D}\left(\frac{\partial \mathbf{U}}{\partial \mathbf{y}}+\frac{\partial \mathbf{V}}{\partial \mathbf{x}}\right)\right]+\frac{\partial}{\partial \mathbf{y}}\left(2 \mathbf{A}_{\mathbf{M}} \mathbf{D} \frac{\partial \mathbf{V}}{\partial \mathbf{y}}\right) \\
& \mathbf{F}_{\boldsymbol{\theta}}=\frac{\partial}{\partial \mathbf{x}}\left(\mathbf{A}_{\mathbf{H}} \mathbf{D} \frac{\partial \boldsymbol{\theta}}{\partial \mathbf{x}}\right)+\frac{\partial}{\partial \mathbf{y}}\left(\mathbf{A}_{\mathbf{H}} \mathbf{D} \frac{\partial \boldsymbol{\theta}}{\partial \mathbf{y}}\right) \\
& \mathbf{F}_{\mathrm{S}}=\frac{\partial}{\partial \mathbf{x}}\left(\mathbf{A}_{\mathbf{H}} \mathbf{D} \frac{\partial \mathbf{S}}{\partial \mathbf{x}}\right)+\frac{\partial}{\partial \mathbf{y}}\left(\mathbf{A}_{\mathbf{H}} \mathbf{D} \frac{\partial \mathbf{S}}{\partial \mathbf{y}}\right)
\end{aligned}
$$

En la Figura 5 se muestra como es el esquema de trabajo del modelo numérico.

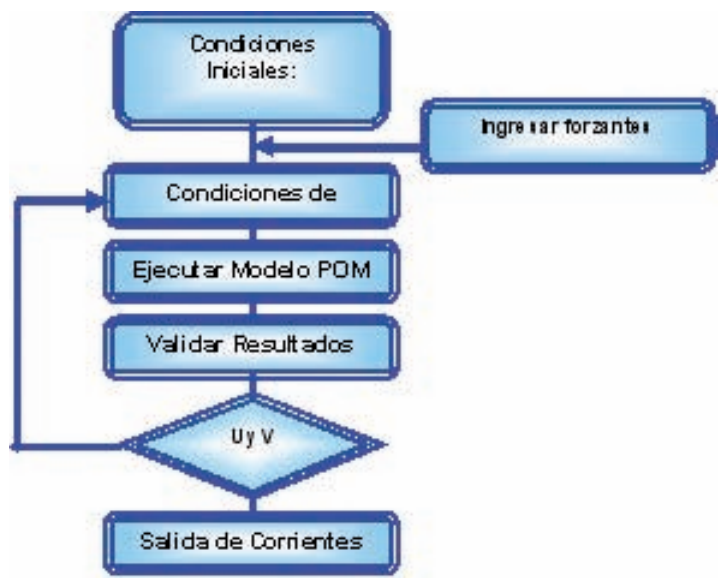

Figura 5. Esquema del funcionamiento del Modelo Numérico 
Actualmente el modelo viene siendo empleado por investigadores en diversos países, así también se tiene disponible una gran cantidad de publicaciones y trabajos empleando dicho modelo numérico.

\section{- Características de la Simulación}

Se empleo una malla de resolución variable y se consideró mayor resolución en la zona del puerto del Callao; sobre esta grilla se interpolaron los datos de batimetría y se trabajó con 6 niveles verticales (niveles sigma)

El modelo comienza a ejecutarse con un inicio de modelación del tipo "Cold Start", donde todas las variables que influyen en la circulación son consideradas nulas en el tiempo cero de simulación, es decir, se parte de un estado de reposo. A partir del inicio de las simulaciones, existe un tiempo de "calentamiento" del modelo, en el cual los resultados del modelo no son tomados en consideración. En este sentido el tiempo de calentamiento que tomó el modelo para este caso fue de 2 días aproximadamente.

En la Tabla 2, se muestran los parámetros considerados.

Tabla 2. Parámetros considerados para la modelación numérica de corrientes marinas.

\begin{tabular}{|l|l|}
\hline \multicolumn{1}{|c|}{ Parámetro } & \multicolumn{1}{c|}{ Valor } \\
\hline Paso de tiempo & 120 segundos \\
\hline$\Delta \times$ Máximo & 500 metros \\
\hline$\Delta$ x Mínimo & 40 metros \\
\hline$\Delta$ y Máximo & 700 metros \\
\hline$\Delta$ y Mínimo & 50 metros \\
\hline Punto de grilla en $X$ & 100 \\
\hline Punto de grilla en $Y$ & 100 \\
\hline Niveles verticales & 6 \\
\hline Tiempo total de simulación & 7 días \\
\hline Inicio de simulación & Cold Star \\
\hline
\end{tabular}

\section{- Condiciones de Frontera}

Las condiciones de frontera consideradas son las siguientes:

Frontera Norte, en esta frontera se fuerza el modelo caracterizando una onda de marea que es representada de la siguiente manera:

$$
\eta=h_{0}+\sum_{1}^{k} h_{r} \cos \left(w_{r} t-\alpha_{r}\right)
$$

Donde:

hr: Amplitud de la componente armónica de marea

wr: Frecuencia de la componente armónica de marea

$\boldsymbol{\alpha}_{\mathbf{r}}$ : Fase de cada componente armónica de marea

ho: nivel del mar, se consideró un valor del nivel del mar de 0.65 metros

Frontera Sur, se consideró un viento de magnitud 6 nudos y una dirección predominante del Sur, además esta condición del viento se mantiene en todos los puntos de grilla.

Frontera Oeste, en esta frontera se considera un gradiente de velocidad nula en la dirección normal a la costa, $\partial \mathbf{U} / \partial \mathbf{x}=0$

Frontera Este, condición de no deslizamiento, de esta manera las componentes de la velocidad son cero en esta frontera.

\section{RESULTADOS Y DISCUSION}

Los resultados de la modelación numérica se indican a continuación en las siguientes graficas:

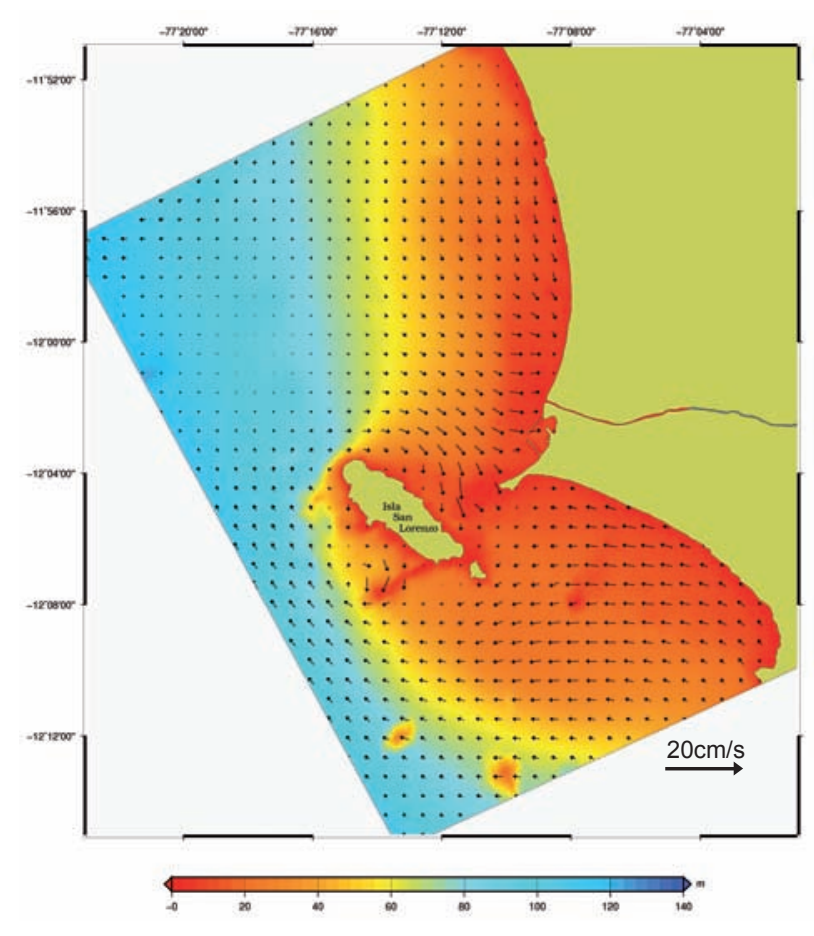

Figura 6. Modelación Numérica de la circulación marina a nivel superficial en marea ascendente para las bahías del Callao y Miraflores. 


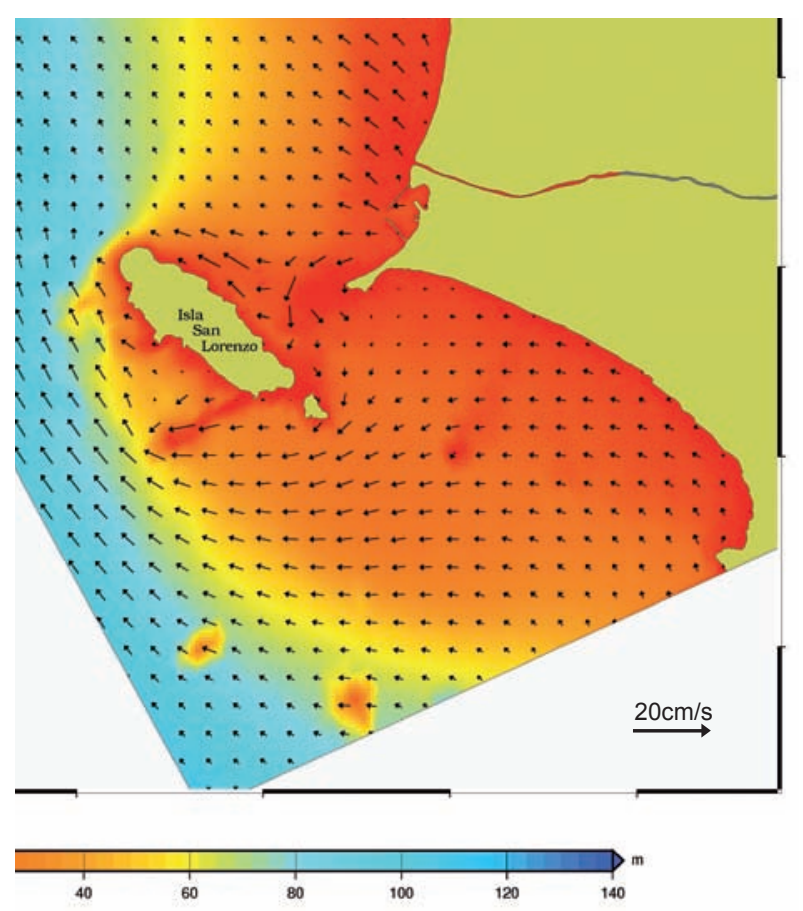

Figura 7. Modelación Numérica de la circulación marina a nivel superficial en marea descendente para las bahías del Callao y Miraflores.

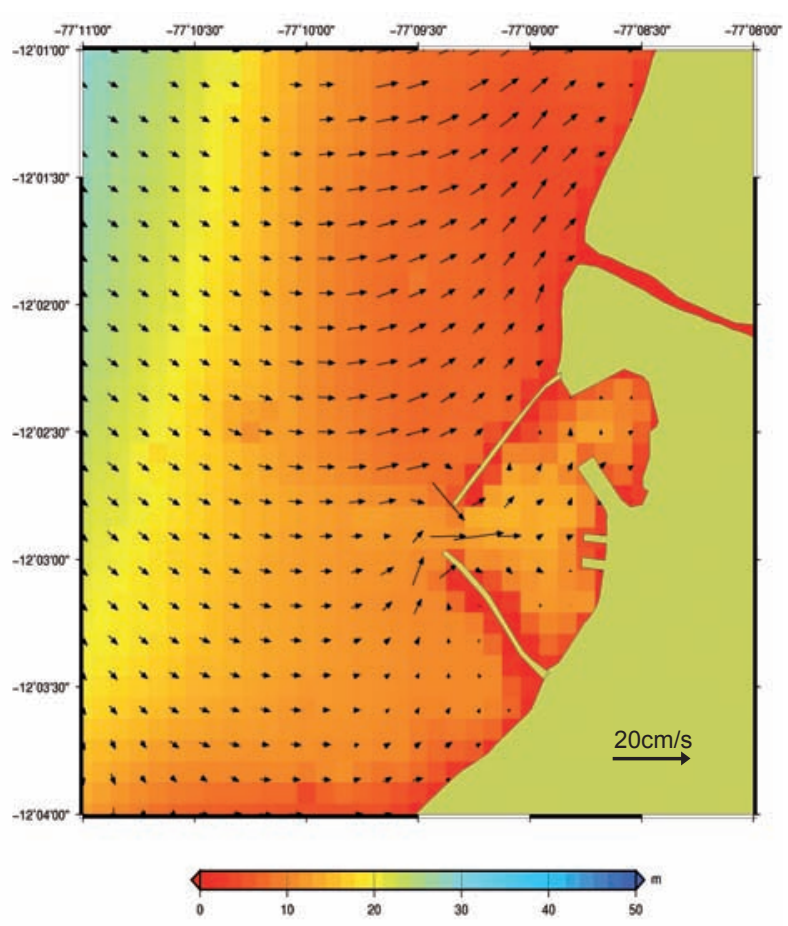

Figura 8. Modelación Numérica de las corrientes marinas a nivel superficial en marea ascendente para la zona del puerto del Callao.

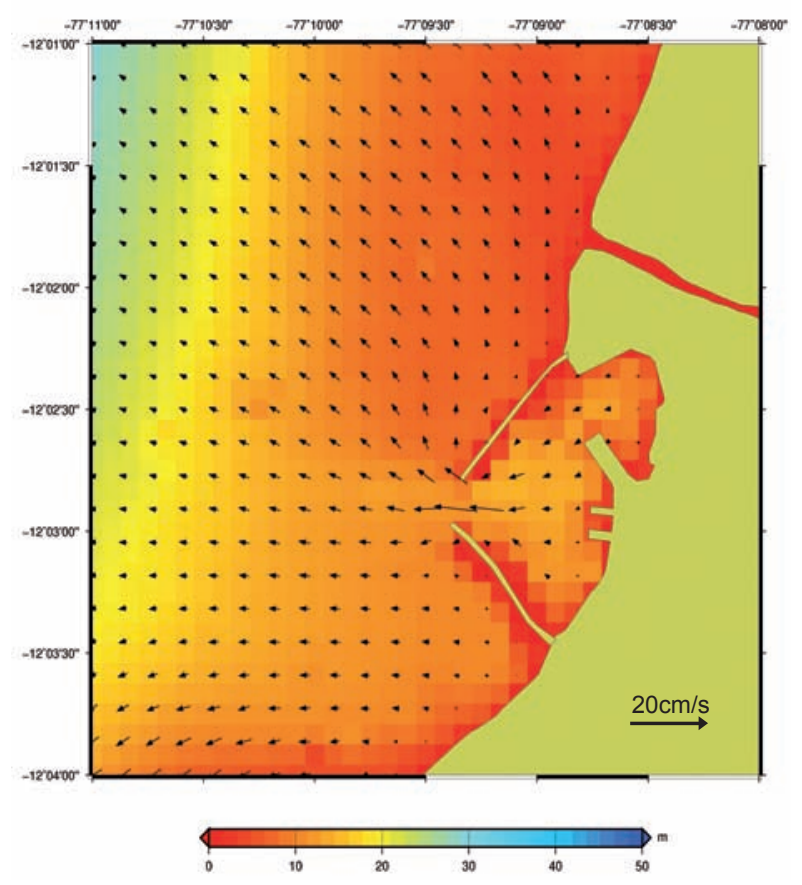

Figura 9. Modelación Numérica de las corrientes marinas a nivel superficial en marea descendente para la zona del puerto del Callao.

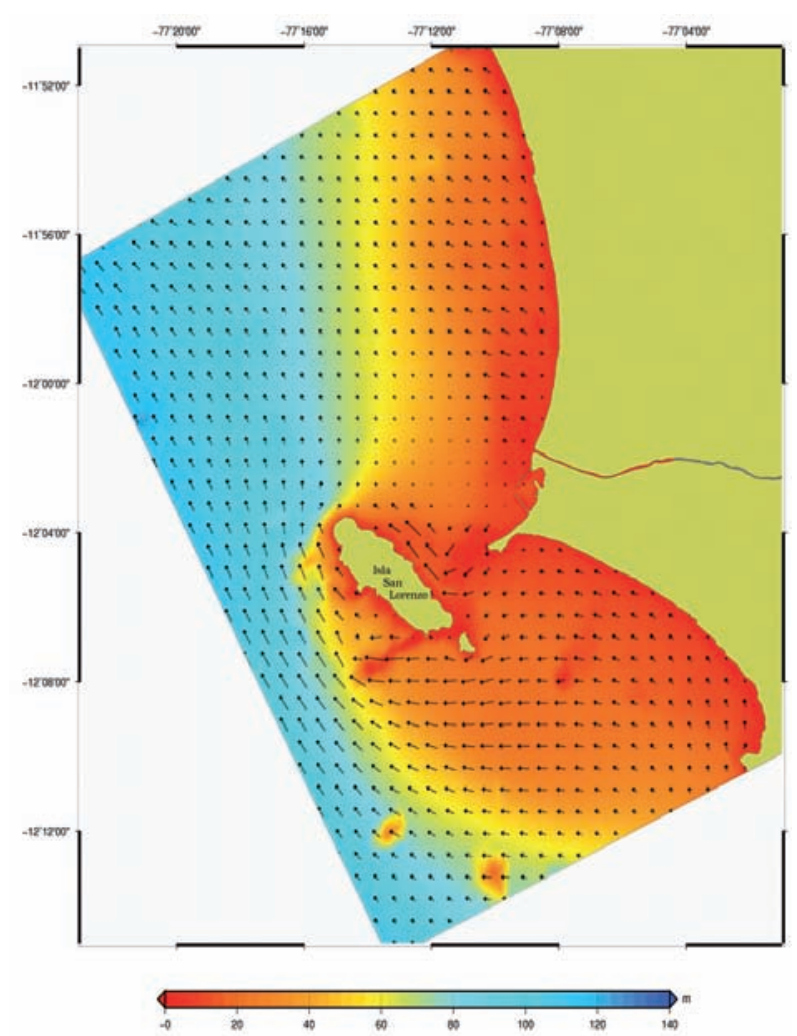

Figura 10. Modelación Numérica de las corrientes marinas a nivel superficial, inicio de la formación de un vórtice en el lado adyacente de la isla. 
De los resultados de la modelación numérica podemos indicar que durante marea ascendente y descendente, las corrientes indican una dirección Norte en la Bahía de Miraflores, luego estas corrientes bordean la isla y se aceleran en el canal natural formado por la Isla y La Punta alcanzando velocidades de hasta $20 \mathrm{~cm} / \mathrm{s}$, ya en la bahía del Callao las corrientes se encuentran con menor velocidad con respecto a la bahía de Miraflores, cabe mencionar que se puede presentar la formación de vórtices en áreas adyacente a la isla.

Para la zona del puerto de Callao se aprecia la influencia de las mareas en la generación de corrientes marinas, indicando una dirección Este para marea ascendente y Oeste para marea descendente, es decir el flujo es entrante y saliente al puerto respectivamente; estas velocidades se ven aceleradas por el estrecho formado por los rompeolas con valores entre 18 a $22 \mathrm{~cm} / \mathrm{s}$, ya dentro del área del puerto esta velocidades disminuyen hasta poder llegar a alcanzar el nivel de aguas tranquilas (velocidades con valores muy pequeños), estos valores son similares a la grafica presentada en la Figura 3.

Haciendo una comparación cualitativa podemos observar que los resultados de la modelación numérica se asemejan a la fotografía satelital indicada en la Figura 4, ello nos estaría indicando que la dirección predominante en aquel momento es hacia el norte para un tipo de marea descendente.

\section{DISCUSIÓN}

De los resultados obtenidos se puede apreciar que existe una marcada variabilidad direccional de las corrientes, que principalmente ingresan a la zona de estudio desde la zona sur de la bahía de Miraflores, acelerándose en el canal natural formado por la Isla San Lorenzo y La punta. En esta parte de la zona de estudios se aprecia la influencia del viento. En la bahía de Callao, el flujo es mas lento, debido a la configuración morfológica de la zona, y en esta zona se pueden apreciar cambios significantes en la dirección de las corrientes marinas, lo que indicaría la influencia de la marea en esta zona, originando los denominados flujos y reflujos. Del mismo modo, se aprecia una constante entrada y salida del flujo dentro de las instalaciones del puerto del Callao. Con estas características de la circulación, es viable el estudio de procesos asociados a la circulación marina como son los fenómenos de dispersión de materiales y de transporte de sedimentos.

\section{CONCLUSIONES}

De los resultados obtenidos, se concluye que la velocidad de las corrientes se ven influenciadas fuertemente por la acción del viento en la bahía de Miraflores ya que no tiene una protección natural como la bahía del Callao que cuenta con la presencia de la Isla San Lorenzo, así también la marea determina la dirección de las corrientes en la zona portuaria, indicando para marea ascendente el ingreso del flujo y para marea descendente la salida del flujo. La presencia de los espigones frena las velocidades a lo largo de este, pero las acelera al momento de ingresar el puerto. Teniendo la base hidrodinámica del área se podrán realizar posteriormente estudios de dispersión por parte de los ríos de la zona, ya que en el presente trabajo que no se están considerando los flujos de agua aportados por estos ríos; así también se podrán realizar estudios de dispersión por parte de descargas industriales, estudios de transporte de sedimentos y estudios para poder observar las variaciones de la circulación cuando ocurra el caso de la construcción de alguna estructura marina.

\section{AGRADECIMIENTOS}

- Escuela Académico Profesional (EAP Ingeniería Mecánica de Fluidos, Universidad Nacional Mayor de San Marcos (UNMSM).

- Grupo de Estudio de la Dinámica Marina (GEDIMA) EAP. Ing. Mecánica de Fluidos, UNMSM.

- Dirección de Hidrografía y Navegación (DHN) de la Marina de Guerra del Perú. Instituto del Mar del Perú (IMARPE).

- DELIMAR SAC. Científicos Marinos.

- Al profesor Lic. Salvador Zuta Rubio de la EAP. Ing. Mecánica de Fluidos, UNMSM.

- A nuestro Asesor Ing. Emanuel Guzmán Zorrilla docente de la EAP. Ing. Mecánica de Fluidos, UNMSM. 


\section{REFERENCIAS}

[1] Estudio de Factibilidad de una Planta de Tratamiento de aguas en Lima.

[2] Estudio de Impacto Ambiental para el Diseño, Construcción, Operación y Cierre del Nuevo Terminal de Contenedores Adyacente al Rompeolas Sur del Terminal Portuario del Callao, ECSA Ingenieros

[3] Oceanografía de las Aguas Costeras del Perú, Salvador Zuta y Oscar Guillén, Instituto del Mar del Perú, Boletín Volumen 2, Número 5, Callao, Perú 1970.
[4] Tesis: Modelamiento de corrientes superficiales inducidas por viento y marea en la bahía Ferrol - Chimbote, Emanuel Guzmán Zorrilla, Lima, Perú 2006.

[5] Mini-Curso proferido no Instituto de Estudos do Mar Almirante Paulo Moreira IEAPM, O Modelo Oceanico da Universidade de Princeton, Prof. Dr. Edmo Campo-Instituto Oceanográfico, Univ. de S. Paulo.

E-mail: miricenteno@gmail.com, ejguzor@gmail.com, apgarciapz@gmail.com. 